\title{
CARACTERIZAÇÃO E ANÁLISE DOS FRAGMENTOS FLORESTAIS E USO DA TERRA NO MUNICÍPIO DE COLARES, NORDESTE DO PARÁ
}

\section{CHARACTERIZATION AND ANALYSIS OF FOREST FRAGMENTS AND LAND USE IN THE MUNICIPALITY OF COLARES, NORTHEAST OF PARÁ}

\author{
Rafaela Rabelo Ferreira da Silva ${ }^{1}$; Thais Gleice Martins Braga ${ }^{2}$; Edivandro Ferreira \\ Machado $^{3}$; Ises Vanderlene Inácio de Souza ${ }^{4}$.
}

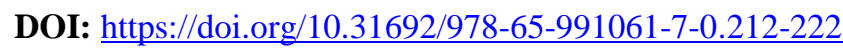

\begin{abstract}
RESUMO
Objetivou-se analisar a cobertura vegetal e uso do solo, assim como caracterizar e analisar os fragmentos florestais presentes na paisagem do município de Colares, através de produtos e técnicas de sensoriamento remoto e geoprocessamento, visando conhecer a estrutura da paisagem de modo a oferecer subsídios ao planejamento de sua ocupação territorial. Para tanto, utilizou-se imagens digitais do sensor OLI/Landsat 8, órbita/ponto 223/061, bandas 4, 5 e 6, as quais foram processadas e classificadas através do algoritmo de máxima verossimilhança. As análises relacionadas à configuração da paisagem e dos fragmentos de vegetação natural foram realizadas com métricas de classe, onde se utilizou o software ArcGis, módulo Patch Analisy. As técnicas de sensoriamento remoto e geoprocessamento permitiram caracterizar e analisar os elementos que compõem a paisagem de Colares, mostrando que esta é definida por um mosaico de cobertura vegetal e uso do solo, evidenciada por uma grande exploração dos recursos naturais, onde a vegetação arbórea encontra-se bastante fragmentada, identificada pela presença de diversos fragmentos pequenos, demonstrando o forte impacto de atividades antrópicas na área. Os resultados mostraram também a presença de fragmentos mais conservados, os quais são importantes para a conservação da biodiversidade, cumprindo importante papel na manutenção da estabilidade da biodiversidade local. Por sua vez, a Associação de Sucessão Secundaria x Culturas Anuais x Pastagem foi a segunda classe de maior predomínio na paisagem, ocupando aproximadamente $15 \%$ (9.033,30 ha) da área do município. Já a classe isolada de Pastagem representou $12 \%$ da paisagem do município, caracterizando uma área de 7.211,70 há.
\end{abstract}

Palavras-Chave: Cobertura Vegetal, Métricas de Classe, Uso do solo.

\section{ABSTRACT}

The study aimed to analyze the vegetation coverage and land use, as well as the characterization and analysis of forest fragments in the landscape of Colares municipality through products and techniques of remote sensing and GIS, improving knowledge of landscape structure to offer subsidies to the planning of its territorial occupation. Indeed, it was used digital images of OLI / Landsat 8, orbit / point 223/061, bands 4, 5 and 6, which were processed and classified using the maximum likelihood algorithm. The analyzes related to the configuration of the landscape and natural vegetation fragments were performed using metric classes, using ArcGIS software, on Patch Analysis module. The use of remote sensing and geoprocessing allowed to characterize and analyze the elements that compose the landscape of Colares, showing that this is characterized by a mosaic of vegetation cover and land use, evidenced by a large exploitation of natural resources, where the arboreal vegetation

\footnotetext{
${ }^{1}$ Bacharelado em Biologia, Universidade Federal Rural da Amazônia, r.rabelo@ outlook.com.br

${ }^{2}$ Mestre em Ciências Ambientais, Museu Paraense Emílio Goeldi, thaisbraga.ambiental@gmail.com

${ }^{3}$ Bacharelado em Biologia, Universidade Federal Rural da Amazônia, edivandro22ferreira@gmail.com

${ }^{4}$ Bacharelado em Biologia, Universidade Federal Rural da Amazônia, ises0souza@gmail.com
} 
is very fragmented, identified by the presence of a large number of small fragments, demonstrating the strong impact of human activity in the area. The results also showed that there is the presence of some more conserved fragments, which are important for the conservation of biodiversity, fulfilling an important role in maintaining the stability of the local biodiversity. In turn, the Association of Secondary Succession x Annual Cultures $\mathrm{x}$ Pasture was the second most prevalent class in the landscape, occupying approximately $15 \%$ $(9,033.30$ ha) of the area of the municipality. The isolated class of Pasture represented $12 \%$ of the landscape of the municipality, characterizing an area of 7,211.70 ha.

Keywords: Vegetation Coverage, Metric Classes, Land Use.

\section{INTRODUÇÃO}

A Amazônia pode ser categorizada como uma região sob grande risco devido à variabilidade e mudança do clima. O risco não se deve apenas à mudança prevista para o clima, mas também às interações sinergéticas com as ameaças existentes não relacionadas às mudanças climáticas, tais como a mudanças do uso e cobertura da terra, fragmentação da floresta e fogo (Marengo, 2009).

A expansão das fronteiras agrícolas, assim como a modernização da agricultura e da pecuária, está entre os fatores que mais contribuem para a descaracterização da vegetação original, impactando, também, a biodiversidade, solos e as águas. De acordo com Valente \& Vettorazzi (2002), o acelerado processo de substituição das paisagens naturais ocorre de fato em função da intensificação do antropismo, normalmente visando o uso do solo, o que por sua vez ocasiona a formação de pequenos fragmentos florestais onde anteriormente existiam áreas de florestas contínuas.

Esta fragmentação florestal, resultante do processo de substituição da cobertura vegetal nativa por áreas de uso antrópico, é a principal causa da perda da biodiversidade, uma vez que contribui para a extinção de espécies (Laurance et al., 2001).

As mudanças no uso do solo, portanto, têm despertado atenção eminente em todo o mundo. Segundo Pimentel (2014), isso se deve ao acelerado processo de mudanças das últimas décadas, assim como aos possíveis impactos ambientais e socioeconômicos que estas mudanças podem acarretar, causando preocupações desde o nível local até o global do meio ambiente e da sociedade.

Assim, o trabalho tem por objetivo analisar a cobertura vegetal e o uso do solo, assim como a caracterização e análise dos fragmentos da vegetação arbórea presente na paisagem do município de Colares no estado do Pará, por meio de produtos e técnicas de sensoriamento remoto e geoprocessamento, visando o conhecimento da estrutura da paisagem de modo a oferecer subsídios ao planejamento de sua ocupação territorial. 


\section{FUNDAMENTAÇÃO TEÓRICA}

A Ecologia da Paisagem vem estudando as relações espaciais e interações entre os fragmentos florestais e também as alterações estruturais das paisagens em diversas escalas, possibilitando a melhor compreensão da dinâmica destas correlações, das funções e das mudanças de uma paisagem. A Ecologia da Paisagem utiliza-se de vários índices e métricas para a caracterização quantitativa, assim podendo comparar paisagens, ou identificar onde se diferenciam. Pode também determinar as relações entre os muitos processos funcionais e os padrões dessas paisagens, uma vez que tais parâmetros possibilitam a quantificação de sua composição e de sua configuração (Turner \& Gardner, 1990).

Assim, preservar a cobertura vegetal de uma área é extremamente importante uma vez que ela é responsável pela proteção física e mecânica do solo, regula a temperatura e a microbiota do solo, protege o relevo, a biodiversidade, o clima, os mananciais e os lençóis freáticos (Mascarenhas et al. 2009). Dessa forma, a vegetação age como um indicador das condições ambientais de uma região, assim como da "saúde" dos ecossistemas.

A mudança da paisagem pode ser parcialmente justificada pelo modelo histórico de ocupação desordenada da Amazônia, através da abertura de estradas e políticas de integração (Cunha \& Almeida, 2002). Devido a esta importância histórica, para entender o desflorestamento na região é importante analisar a conjuntura do desmatamento, verificando por meio de imagens de satélite classificadas em classes de uso e ocupação do solo para entender a dinâmica de uso da terra após o desflorestamento. Dessa forma, segundo Fearnside (2006), os impactos que possam vir a ocorrer, dentre os que já são perceptíveis, incluem a perda de oportunidades para o uso sustentável da floresta, assim como a produção de mercadorias tradicionais tanto por manejo florestal de madeira como por extração de produtos não-madeireiros.

\section{METODOLOGIA}

Esta pesquisa é de base quali-quantitativa, por meio da qual se busca compreender a fragmentação florestal e o uso da terra no município de Colares e pode se caracterizada como sendo do tipo estudo de caso, pois busca, além de informações teóricas, observações, experiências e coletas de dados. Este trabalho foi desenvolvido no município de Colares, que está localizado na Mesorregião do Nordeste Paraense.

Como suporte cartográfico, foi utilizada a base planialtimétrica compilada a partir do uso de dados digitais disponibilizados pelo IBGE, na escala de 1:100.000, contendo entre outros elementos, a rede de drenagem e a malha viária existente da área de estudo. Já para o 
levantamento da cobertura vegetal e do uso do solo, foi empregada imagem orbital obtida pelo sensor OLI a bordo do satélite LANDSAT-8. A imagem selecionada refere-se à órbita/ponto 223/061, bandas TM 6, 5 e 4, do ano de 2013, adquirida a partir da página eletrônica da Science for a changing world.

Softwares empregados no desenvolvimento do presente trabalho foram o Environment For Visualizing Images - Envi 4.5 (ENVI, 2011) para o tratamento e processamento digital das imagens de satélite; e o software ArcGis 9.3 (ESRI, 2010) para a análise da estrutura da paisagem e confecção dos mapas. No auxílio à navegação em tempo real com o GPS em campo, e na descarga dos pontos com coordenadas geográficas coletadas in loco, foi empregado o GPS Trackmaker Free V.13.0.

$\mathrm{Na}$ fase de reconhecimento da paisagem em estudo, utilizou-se a técnica de navegação em tempo real sobre a carta imagem, onde se acoplou um GPS de navegação, modelo GPSmap 60CSx, ao Notebook da marca e Machines E725-4122, com o sistema operacional Windows XP professional sp2, visando o uso da ferramenta 'simulação', do software GPS TrackMaker free v.13,0. Foram feitos registros fotográficos utilizando uma câmera Digital Canon A480 de 10MP de resolução, ressaltando que os mesmos somaram 110 fotos de locais representativos dos elementos da paisagem do município de Colares. Foi feita incursão em campo com o objetivo de correlacionar as diversas feições espectrais presentes nas imagens Landsat-8 com os padrões de cobertura vegetal e uso do solo observado in loco. Ja as análises relacionadas à configuração da paisagem e dos fragmentos de vegetação natural foram realizadas por meio do software ArcGis, módulo Patch Analisy.

\section{RESULTADOS E DISCUSSÃO \\ CARACTERIZAÇÃo dA COBERTURA VEGETAL E USO DO SOLO DO MUNICÍPIO DE COLARES}

É possível avaliar por meio da Figura 1 e da Tabela 1 as classes de cobertura vegetal e uso do solo presentes no município de Colares, bem como suas respectivas áreas de abrangência. É possível observar que a área ocupada pela classe de Vegetação Arbórea (representada neste estudo pela Floresta Ombrófila Densa e vegetação sucessional em estágios avançados), foi de 12.532,68 ha, cerca de $20 \%$ de toda paisagem (Tabela 1), está presente predominantemente ao longo dos rios, caracterizando-se com Áreas de Preservação Permanente. 
Figura 1: Mapa de Cobertura Vegetal e Uso do Solo do Município da Ilha de Colares-PA. Fonte: Autores.

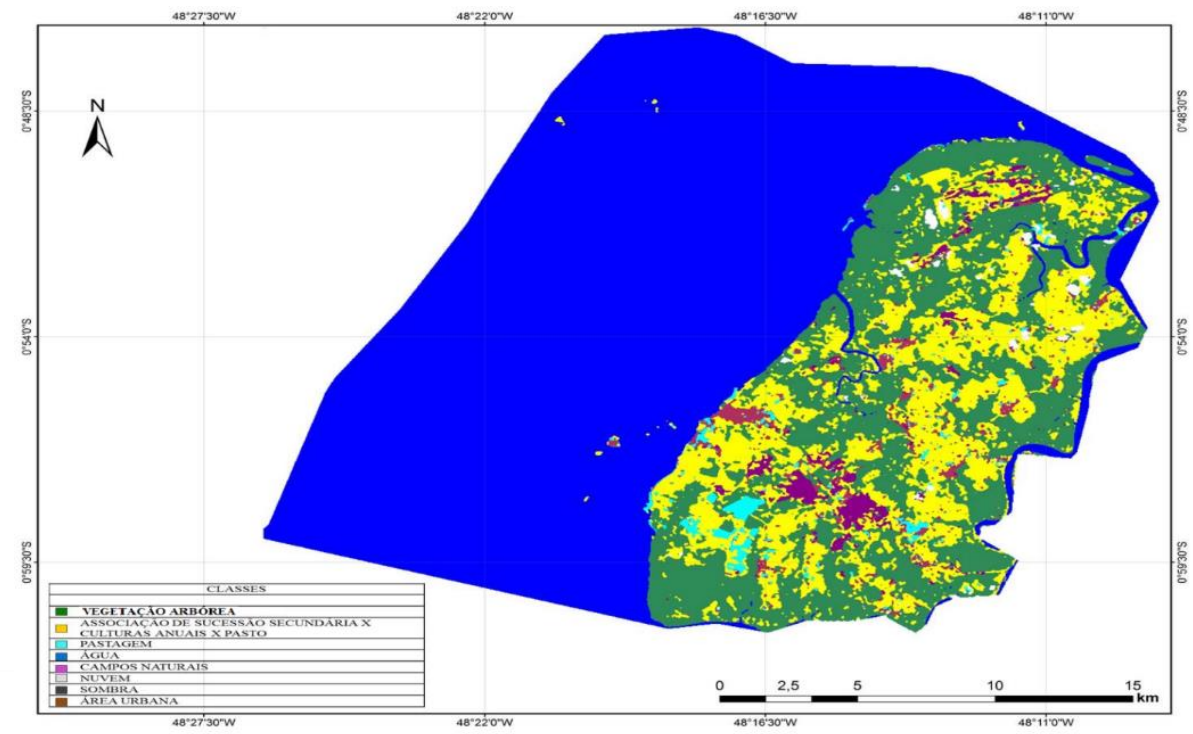

Tabela 1: Área e percentual ocupado pelas classes de cobertura vegetal e uso do solo do município de Colares Pará. (Área em há e \%). Fonte: Autores.

\begin{tabular}{c|cc}
\hline CLASSES & ÁREA (ha) & \% \\
\hline Vegetação Arbórea & $12.432,68$ & 20,53 \\
Associação de Sucessão Secundária x Culturas & $9.033,30$ & 14,00 \\
Anuais x Pastagem & $7.211,70$ & 11,91 \\
Pastagem & 878,49 & 1,45 \\
Campos Naturais & 834,93 & 1,38 \\
Área Urbana/Solo Exposto & $37.171,80$ & 51,00 \\
Água & 84,06 & 0,12 \\
Nuvem & 13,80 & 0,02 \\
Sombra &
\end{tabular}

A Associação de Sucessão Secundaria x Culturas Anuais x Pastagem foi a segunda classe de maior predomínio na paisagem, ocupando aproximadamente 15\% (9.033,30 ha) da área do município. Esta classe configura o tipo de agricultura praticado na região, uma vez que, segundo Denich (1991), o sistema de produção da pequena agricultura constitui-se, essencialmente, de dois subsistemas consecutivos: a fase de cultivo com culturas alimentares de interesse econômico e da vegetação secundária como fase de pousio, sendo representada tradicionalmente pela agricultura de pequeno e médio porte. Segundo IBGE (2007), a agricultura praticada no Nordeste Paraense ainda apresenta como base predominante o sistema de subsistência, o qual se engloba num nível de evolução econômica incipiente que envolve entre seus principais produtos: o feijão-caupi (Vigna unguiculata), a mandioca (Manihot esculenta L.), o milho (Zea mays L.), o dendê (Elaeis guineenses Jacq.) e a pimentado reino (Piper nigrum L.). 
Assim, para o mesmo autor, a vegetação secundária desempenha nos regimes da pequena exploração agrícola, a função de acumular na fitomassa os nutrientes a serem liberados através da queima, para permitir a fase de cultivo subseqüente, além de ser importante no controle das ervas daninhas instaladas durante a fase de cultivo anterior.

A classe isolada de Pastagem representou $12 \%$ da paisagem do município, caracterizando uma área de 7.211,70 ha, indicando que), demonstrando a afinidade da região com a pecuária, a presença de grandes áreas de pastagens é uma realidade observada em todo o nordeste Paraense, evidenciada em diversos estudos, entre eles: Almeida et al. (2006); Watrin et al. (2007); e Watrin et al. (2009). Este padrão é observado para toda a Amazônia, onde as áreas de pastagem cultivada constituem o uso de terra dominante.

Observa-se que metade da área do município é composta pela classe Água, identificando-se também a presença de Campos Naturais abrangendo 1,45\% do município (aproximadamente 880 ha), os quais se encontram dispostos em padrão de faixas de áreas inundadas que segundo Pires (1995), constituem excelentes corredores ecológicos em uma paisagem.

É possível observar através da dinâmica de mudança de uso da terra no município de Colares, entre os anos de 2004 e 2014 que há uma mudança considerada no uso da terra. Também é observado um ganho de área significativo para as classes de Vegetação Secundária (1256,55 ha), Área não Observada, Área Urbana, e Mosaico de Ocupações (Figura 2 e 3).

Figura 2. Classes de uso do solo no município de Colares em 2004. Fonte: Autores.

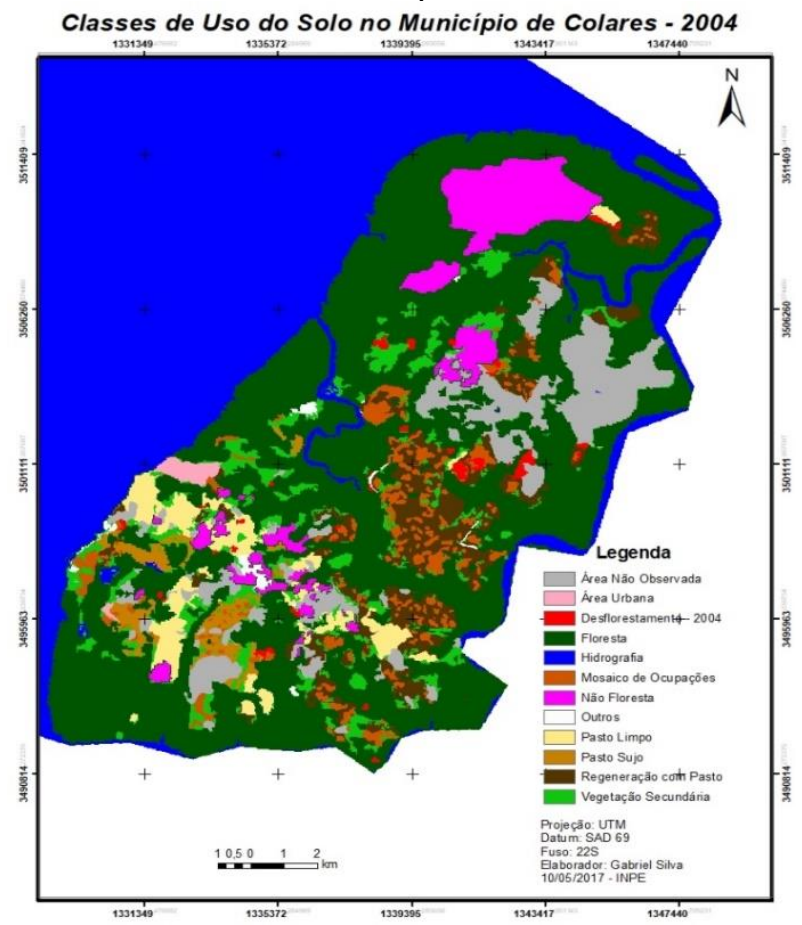


Figura 3. Classes de uso do solo no município de Colares em 2014. Fonte: Autores.

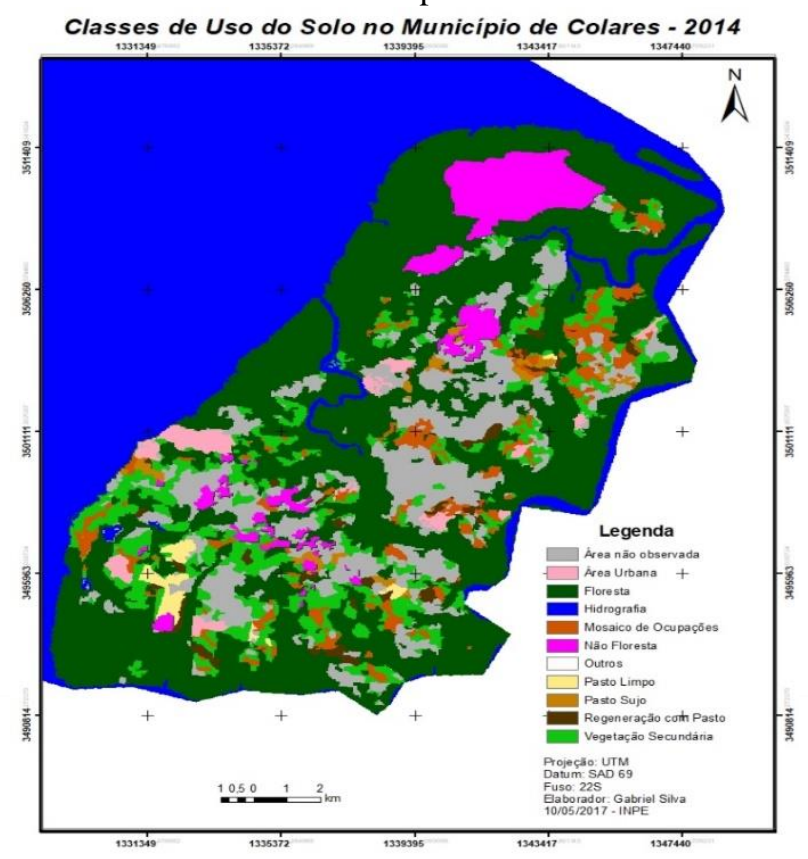

\section{CARACTERIZAÇÃo E ANÁLISE DOS FRAGMENTOS DE VEGETAÇÃo ARBÓREA DA PAISAGEM DO MUNICÍPIO DE COLARES}

A caracterização e análise da vegetação arbórea da paisagem foram feitas por meio de métricas da paisagem, as quais quantificam a composição e/ou a configuração da paisagem que afetam de forma significativa os processos ecológicos, tanto independente quanto simultaneamente. As métricas referentes à vegetação arbórea do município de Colares podem ser verificadas na Tabela 2 a seguir.

Tabela 2. Valores das métricas dos fragmentos de vegetação arbórea do município de Colares. Fonte: Autores.

\begin{tabular}{c|c}
\hline MÉTRICAS & VALORES \\
\hline Área total (ha) & $12.670,06$ \\
\hline $\mathrm{N}^{\mathrm{o}}$ de Fragmentos & 270,70 \\
\hline Tamanho médio de fragmentos (ha) & 46,93 \\
\hline Total de borda (m) & $1.124 .851,98$ \\
\hline Tamanho médio de borda (m) & 4135,48 \\
\hline Dimensão fractal média & 1,38 \\
\hline Área total de interior (ha) & $3.289,77$ \\
\hline $\mathrm{N}^{\circ}$ de áreas de interior & 190,00 \\
\hline
\end{tabular}

Observa-se por meio da Tabela 2 que a paisagem do município de Colares é bastante fragmentada, sendo esta formada por 270 fragmentos de Floresta, com tamanho médio de 46,93 ha.

Segundo Casimiro (2000) o número de manchas de um determinado habitat pode influenciar uma grande variedade de processos ecológicos, como por exemplo, a 
determinação do número de subpopulações de uma população espacialmente dispersa, pode alterar a estabilidade das interações e oportunidades de coexistência em sistemas de predador - presa e sistemas competitivos. Por outro lado, quanto maior a subdivisão da paisagem, maior número de manchas, maior a resistência potencial à propagação de perturbações (doenças, fogo), podendo as manchas persistirem mais facilmente do que se o número fosse diminuto.

As bordas dos fragmentos compreendem $1.124 .851,98 \mathrm{~m}$, o que enfatiza o elevado grau de fragmentação do município, pois quanto maior for o total de margens, maior a fragmentação da paisagem, assim sendo a quantidade total de margens é um excelente indicador da configuração da paisagem. A importância das margens é muito relevante, pois há uma importante interação da flora e da fauna nestas áreas de margem, que podem constituir habitats por sí só, além de serem claramente frentes de interface: predação e refúgio, dispersão de sementes e re-colonização, proteção do vento, etc. (O'Neill, 1988).

A dimensão fractal média apresentada para tais fragmentos foi de 1,38, denotando que tais fragmentos apresentam pouca complexidade em suas bordas, tendendo a apresentar formas regulares, o que evidencia a forte presença humana na paisagem do município. A forma dos fragmentos florestais é um importante parâmetro a ser considerado, pois está diretamente relacionado ao efeito de borda, determinando o grau com que esse efeito age sobre o fragmento e a maior ou menor influência dos fatores externos sobre a sua biodiversidade (Pires, 1995).

Haja vista, para efetuar a análise do efeito de borda, considerou-se uma margem de borda de $100 \mathrm{~m}$. Assim sendo, os resultados mostram uma área total de interior dos fragmentos de 3.289,77 ha, o que representou apenas $26 \%$ da área de fragmentos. Assim sendo, pode-se afirmar que $74 \%$ da área dos fragmentos de vegetação natural que compõem o município são constituídas por ambiente de borda, o que evidencia a presença de fragmentos pequenos e/ou alongados, tornando a paisagem da mesma prejudicada quanto à qualidade do hábitat, o qual fica mais exposto aos efeitos de borda. $\mathrm{O}$ efeito de borda é um dos mais importantes efeitos causados pela fragmentação de hábitats.

As áreas de interior dos fragmentos apresenta um valor de 3.289,77 ha o que segundo Metzger (1997), não é suficiente para manter a sustentabilidade de algumas espécies e a integridade de sua estrutura natural, pois o mínimo necessário pra isso seria uma área de aproximadamente 25.000 ha. O estabelecimento de uma estrutura interna está, portanto, relacionada a uma área mínima, capaz de manter as espécies típicas do tipo de formação florestal a que o fragmento pertence. 
Embora o processo de fragmentação possa ocorrer naturalmente em função da ação do tempo acrescida dos intemperismos, a ação antrópica, neste caso, vem atuando decisivamente no sentido de acelerar e desestabilizar o fluxo natural das transformações nos ecossistemas locais. Para Bender et al. (1998), nos casos em que a fragmentação cria um grande número de fragmentos a partir de um sistema contínuo, as espécies do interior normalmente podem sofrer um declínio em sua população, pois estarão atuando em conjunto os efeitos do tamanho do fragmento e da perda de habitat. Este padrão continuará com o declínio do tamanho do fragmento, até que todo o fragmento seja constituído inteiramente por habitats com características de borda.

\section{CONCLUSÕES}

O emprego de produtos e técnicas de sensoriamento remoto e de geoprocessamento permitiram realizar a caracterização e a análise dos elementos estruturais da paisagem do município de Colares-PA, por meio da avaliação quantitativa e qualitativa dos fragmentos de vegetação arbórea encontrados na área do mesmo.

O município de Colares é caracterizado por um mosaico de classes de cobertura vegetal e uso da terra, evidenciando uma grande exploração dos recursos naturais, onde a vegetação arbórea está presente predominantemente ao longo dos cursos d'água, caracterizando-se como Áreas de Preservação Permanente.

A paisagem do município de Colares encontra-se bastante fragmentada, caracterizada pela presença de um grande número de fragmentos pequenos, demonstrando o forte impacto da atividade humana na área.

Os fragmentos de vegetação arbórea demonstraram uma tendência de aumento do índice de dimensão fractal com o aumento da área. Dessa forma, os fragmentos maiores tenderam a possuir formas mais complexas, ficando assim mais expostos aos efeitos de borda.

Constatou-se que a paisagem do município encontra-se fortemente comprometida no que tange a integridade ecológica, uma vez grande parte da sua área de vegetação arbórea (74\%) é constituída de ambiente de borda, fator que afeta significativamente a qualidade dos ecossistemas ali existentes.

A presença de alguns fragmentos mais conservados, com expressivas áreas centrais, constitui-se de fundamental importância para a conservação da biodiversidade na área de estudo, apesar de não serem em grande número, cumprindo um importante papel na manutenção da estabilidade da biodiversidade local. 


\section{REFERÊNCIAS}

ALMEIDA, E.; SABOGAL, C.; BRIENZA JÚNIOR, S. Recuperação de Áreas Alteradas na Amazônia Brasileira: Experiências Locais, Lições Aprendidas e Implicações para Políticas Públicas. Belém: CIFOR, 2006.

BENDER, D. J.; CONTRERAS, T. A.; FAHRIG, L. Habitat loss and population decline: a metaanalysis of patch size effect. Ecology. v.79, n. 2, p.517-533, 1998.

CASIMIRO, Pedro Cortesão. Uso do solo - Ecologia da paisagem, perspectiva de uma nova abordagem no estudo da paisagem em geografia. GeoInova, n. 2, DGPR - FCSH - UNL, Lisboa, p. 45-65, 2000.

CUNHA, Manuela Carneiro da; ALMEIDA, Mauro Barbosa de. Enciclopédia da floresta: o Alto Juruá: práticas e conhecimentos das populações. São Paulo: Companhia das Letras, 2002. $784 \mathrm{p}$.

DENICH, M. A Importância de uma Vegetação Secundaria Nova para o Incremento da Produtividade do Sistema de Produção na Amazônia Oriental Brasileira. EMBRAPA. CPATU-GTZ, Belém. 1991. 284p.

ENVIRONMENTAL SYSTEMS RESEARCH INSTITUTE (ESRI). Guia do ArcGis Disponível em: <http://www.esri.com/products/index.html> Acesso em: dez 2010.

FEARNSIDE, Philip M.. Desmatamento na Amazônia: dinâmica, impactos e controle. Acta Amaz., Manaus, v. 36, n. 3, p. 395-400, 2006.

IBGE, Instituto Brasileiro de Geografia e Estatística, diretoria de geociências, mapastematicos_pedologia/unidades_federação/pa_pedologia, 2007.

LAURANCE, W. F., M. A. COCHRANE, S. BERGEN, P. M. FEARNSIDE, P. DELAMÔNICA, C. BARBER, S. D'ANGELO, AND T. FERNANDES. 2001. The future of the Brazilian Amazon: development trends and deforestation. Science, 291:438-439.

MARENGO, J. A.; NOBRE, C. A.; BETTS,R,A.; COX, P, M.; SAMPAIO, G.; SALAZAR, L; Aquecimento Global e Mudança Climática na Amazônia: Retroalimentação ClimaVegetação e Impactos nos Recursos Hídricos. Amazonia and Global Change Geophysical Monograph Series 186. 2009.

MASCARENHAS, L. M. A. et al. 2009. Sensoriamento remoto como instrumento de controle e proteção ambiental: análise da cobertura vegetal remanescente na Bacia do Rio Araguaia. Sociedade \& Natureza, 21(1): 5-18.

Metzger, J.P. \& Décamps, H. 1997. The structural connectivity threshold: an hypothesis in conservation biology at the landscape scale. Acta Ecologica 18: 1-12.

O'NEILL, R. V.; MILNE, B.T.; TURNER, M.G. \& GARDNER, R.H. Resource utilization scales and landscape pattern. Landscape Ecology, v.2. p. 63-69, 1988.

PIMENTEL, T. R. G.; Classificação de padrões temporais de uso do solo e cobertura da terra em séries temporais de índice de vegetação utilizando um sistema neuro-difuso. 
Dissertação (Mestrado em Computação Aplicada) - Instituto Nacional de Pesquisas Espaciais, São José dos Campos, 2014.

PIRES, J.S.R. Análise Ambiental voltada ao Planejamento e Gerenciamento do Ambiente Rural: Abordagem Metodológica aplicada ao Município de Luiz Antônio - SP. Tese de Doutorado. Universidade Federal de São Carlos, São Carlos, São Paulo, 1995. 164p.

PEREIRA, B.W.F.; Análise da estrutura da paisagem da microbacia do rio Peixe-Boi, Nordeste Paraense, com base na fragmentação da vegetação arbórea - PA. Dissertação Universidade Federal Rural da Amazônia, 2012. 82 f.

TURNER, M. G.; GARDNER, R. H. Quantitative methods in landscape ecology: the analysis and interpretation of landscape heterogeneity. New York: Springer Verlag, 1990. $536 \mathrm{p}$.

VALENTE, R. O. A.; VETTORAZZI, C. A. Análise da estrutura da paisagem na Bacia do Rio Corumbataí, SP. Scientia Forestalis, n.62, p.114-129, 2002.

WATRIN, O. S.; MACIEL, M.N.M.; THALÊS, M.C. Análise espaço-temporal do uso da terra em microbacias hidrográficas no município de Paragominas, Estado do Pará. In: SIMPÓSIO BRASILEIRO DE SENSORIAMENTO REMOTO (SBSR), Florianópolis. 2007. Anais... SP: INPE, 2007. p.7019-7026. CD-ROM.

WATRIN, O. S.; GERHARD, P.; MACIEL, M.N.M. Dinâmica do uso da terra e configuração da paisagem em antigas áreas de colonização de base econômica familiar, no nordeste do estado do Pará. Geografia, v.34, n. 3, p.455-472, set/dez. 2009. 